\title{
Interaction with the nuclear matrix of a chimeric construct containing a replication origin and a transcription unit
}

\author{
Jacques N. Adom ${ }^{1}$, Fabrice Gouilleux ${ }^{2}$ and Hélène Richard-Foy \\ Unité de Recherches sur les Communications Hormonales, INSERM U-33, Hôpital du Kremlin Bicêtre, Bicêtre (France)
}

(Received 31 March 1992)

(Revised manuscript received 14 August 1992)

\begin{abstract}
Key words: Nuclear matrix; Origin of replication; Transcription activation; Bovine papilloma virus; Harvey murine sarcoma virus;
\end{abstract} Mouse mammary tumor virus

\begin{abstract}
We have studied the interaction of a chimeric construct containing an origin of replication (from bovine papilloma virus) and a hormonally regulated transcription unit (long terminal repeat from the mouse mammary tumor virus, driving the v-Ha-ras gene) with the nuclear scaffold and matrix from mouse fibroblasts. We used two experimental approaches because the nuclear matrix protein composition depends largely on the isolation conditions, making its definition mostly operational. In situ studies and in vitro experiments performed in 1361.5 cells, a cell line in which multiple copies of the construct have been established, indicate that two interesting regions of the construct interact with the nuclear matrix. The first region is located in the v-Ha-ras gene 5 '-flanking sequences. These sequences come from the Harvey virus and contain a piece of the virus like 30S (VL30) sequences in which the v-Ha-ras gene is embedded. This DNA fragment was coupled to the thymidine kinase (TK) promoter driving the reporter luciferase gene and assayed in transient transfection experiments. Its insertion, in the sense orientation, upstream of the TK promoter resulted in a moderate enhancement (2-3-fold) of the luciferase activity. The second region is the most interesting from a physiological point of view. It contains the plasmid maintenance sequence 1 (PMS-1) and the core origin of replication of the bovine papilloma virus. Differences in the results from in situ (nuclear scaffold) and in vitro (nuclear matrix) experiments suggest that the components involved in the interaction with PMS-1 and the viral origin of replication are different. This may be of importance in the context of the recently proposed view that PMS-1 could be part of a composite origin of replication and provide information at a distance.
\end{abstract}

\section{Introduction}

The compaction of long eukaryotic DNA in the nuclei is achieved through its organization into chromatin fiber. In addition, cytological and molecular studies suggest a structural subdivision of eukaryotic DNA into topologically defined domains forming loops [1-4]. Loop formation is thought to result from the anchorage of the chromatin fiber to an internal nuclear structure, termed nuclear 'matrix', 'scaffold', 'skeleton', or 'cage', depending on the authors. The nuclear matrix is a proteinaceous nuclear substructure that can be isolated by sequential treatment of nuclei with nonionic detergents, nucleases, and high-salt concentrations [5-8], or by low salt extraction [9].

\footnotetext{
Correspondence to: H. Richard-Foy, Lab. Hormones, 94276 Bicêtre Cedex, France.

${ }^{1}$ Present address: Département de Biochimie, Faculté des Sciences et Techniques, Abidjan, Côte d'Ivoire.

2 Present address: Friedrich Miescher Institute, Basel, Switzerland.
}

A role for the nuclear matrix in dynamic processes, such as chromatin organization (for a review see Ref. 10), DNA replication [11-14], gene transcription [1517], or RNA processing [18] is increasingly proposed. The nuclear matrix may influence these processes either by increasing local concentrations of available regulatory factors and enzymes, or through the looping mechanism, generating topologically constrained domains, or both. Nuclear matrix may ensure the structural compartmentalization of the nuclei and provide the surface necessary for the organization of multienzymatic complexes involved in gene expression [19].

DNA sequences that exhibit specific interactions with the nuclear matrix (MARs) and scaffold (SARs) have been identified within defined genes (Refs. $8,9,11,19,21$ and 22 for a review). They most frequently map next to regions containing sequences functioning in vivo as transcriptional enhancers or at the boundaries of active chromatin domains, suggesting that such an interaction may delineate both structural and functional domains. MARs/SARs display characteristic features; they are AT rich sequences from 300 to 
approx. $1000 \mathrm{bp}$ in length and usually contain several consensus sequences for Drosophila topoisomerase II $[9,20,22]$. In addition other type of sequences, mostly present within transcriptionnaly active domains have also been described $[23,24]$. Furthermore, it has been pointed out that different methods of analysis detect a somewhat different spectrum of matrix-attached DNA fragments [25,26]. The protein composition of the nuclear matrix is complex and varies considerably depending on the isolation procedure. Topoisomerase II, which plays a critical role at the end of DNA replication in chromosome segregation, has been identified as a major component of the metaphase chromosomal scaffold [27-29]. More recently, two proteins able to generate in vitro looping of the DNA, RAP-1 [30] and ARBP [31] have been purified on the basis of their affinity for MARs/SARs.

The chromatin structure of the glucocorticoidregulated mouse mammary tumor virus long terminal repeat (MMTV LTR) promoter has been described previously [32]. Studies were performed in cell lines containing permanently established DNA chimeric constructs comprising a hormonally regulated transcription unit (MMTV LTR driving the v-Ha-ras reporter gene) linked to the $69 \%$ bovine papilloma virus (BPV) transforming region, used as a eukaryotic vector [33]. In these cell lines the chimeric constructs fractionate with the active chromatin, whether the hormone is present or not (H. Richard-Foy, unpublished observation), suggesting that it has an active or 'poised' chromatin structure [34] and that one or several regions of the construct may interact with the nuclear matrix.

In the work reported here we have used one of these cell lines (1361.5), which has 200 copies of the chimeric construct tandemly integrated in the genome to investigate the interaction with the nuclear scaffold and matrix of regions of the chimera implicated in dynamic processes such as transcription or replication. In order to minimize effects of the method of analysis two approaches have been used: in situ experiments on 'stabilized' nuclei and in vitro studies. One DNA fragment, which does not display a typical SAR/MAR structure, located in the construct immediately up- stream of the v-Ha-ras coding sequences, was found to interact with both nuclear scaffold and matrix and its role on transcription was therefore assayed in transient tranfection experiments.

\section{Materials and Methods}

\section{Tissue culture and transfections}

Two cell lines were used in this work: NIH-3T3 and 1361.5 (NIH-3T3 cells transformed by transfection with the chimeric construct pM23 (Fig. 2B) in which the PBR322 sequences were deleted). This construct contains the $69 \%$ transforming fragment of the BPV1 genome and the mouse mammary virus long terminal repeat (MMTV LTR) driving the v-Ha-ras gene [33]. Cells were maintained in Dulbecco's modified Eagle's medium supplemented with $10 \%$ fetal calf serum. For transfection experiments, NIH-3T3 cells were plated to a density of $5 \cdot 10^{5}$ cells $/ 10 \mathrm{~cm}$ dish. Medium was changed $3 \mathrm{~h}$ before transfection. Each dish was transfected with: $2-5 \mu \mathrm{g}$ of the plasmid to be tested, $5 \mu \mathrm{g}$ of control plasmid ( $\mathrm{pCH} 110$ ), and total DNA adjusted to $20 \mu \mathrm{g}$ with plasmid pSP72. DNA samples were mixed and coprecipitated with calcium phosphate according to standard procedure [35]. $16 \mathrm{~h}$ after transfection, plates were rinsed twice with DMEM without serum, and the medium was changed. Cells were collected 24 $\mathrm{h}$ later. Each construct was assayed in at least three independent transfections.

\section{Luciferase and $\beta$-galactosidase assays}

Cell extracts for luciferase and $\beta$-galactosidase assays were prepared according to Van Trung Nguyen et al. [36] with the modifications previously described [37]. Luciferase and $\beta$-galactosidase activities were measured as previously described [37]. To correct for differences in transfection efficiencies, the luciferase data were normalized to the $\beta$-galactosidase data in each individual sample. Normalized results are expressed in arbitrary units and represent the ratio luciferase activity (RLU (integration time $30 \mathrm{~s}, 50 \mu \mathrm{l}$ sample)) $/ \beta$ galactosidase activity $\left(A_{420}\right.$ (reaction time $2 \mathrm{~h}, 200 \mu \mathrm{l}$ sample)).

\footnotetext{
Fig. 1. In situ interaction of the chimeric construct with the nuclear scaffolds in 1361.5 cells. A map of the chimeric construct established in 1361.5 cells is presented on top left (see Materials and Methods). Genetic elements are indicated inside the circle (HMSV, sequences from Harvey murine sarcoma virus; $\mathrm{p} 21$, sequences coding for the $\mathrm{v}$-Ha-ras protein; Tn9CAT, part of the Tn 9 transposon containing chloramphenicol acetyl transferase coding sequences; SV40, part of SV40, containing a splice and a polyadenylation site; BPV, $69 \%$ transforming fragment of the bovine papilloma virus; PBR, PBR322; INS is the extraneous $1.2 \mathrm{~kb}$ DNA fragment acquired by the construct). The arrows on the circle mark Hae III cutting sites. The regions labelled A, B and C. correspond to the probes used in the experiments presented in panels A, B and C, respectively. Probe A spans from Eco RI to HindIII (positions 7946 to 11045), probe B fron Bam HI to Eco RI (positions 4342 to 7946 ) and probe $\mathrm{C}$ from ClaI to $\mathrm{XbaI}$ (positions 374 to 2953). Nuclei from 1361.5 cells were first extracted with the indicated concentrations of LIS, and then the DNA was cleaved with Haelll. After purification $10 \mu \mathrm{g}$ of each DNA sample was loaded on triplicate gels. The DNA fragments, released (S) or remaining attached to the nuclear matrix $(\mathrm{P})$, were identified by Southern blotting. M: size marker. T: untreated total 1361.5 cellular DNA cut with Haelll. C1, C2 and $\mathrm{C} 3$ show total nuclear DNA from the following controls: C1, nuclei untreated with LIS and digested with HaellI; C2, nuclei treated at $37^{\circ} \mathrm{C} ; \mathrm{C} 3$, nuclei treated with $25 \mathrm{mM}$ LIS. Panel A: probe A; panel B: probe B; panel C: probe C. DNA fragments interacting with the nuclear scaffolds are marked with an arrow and shown by bold lines on the map.
} 

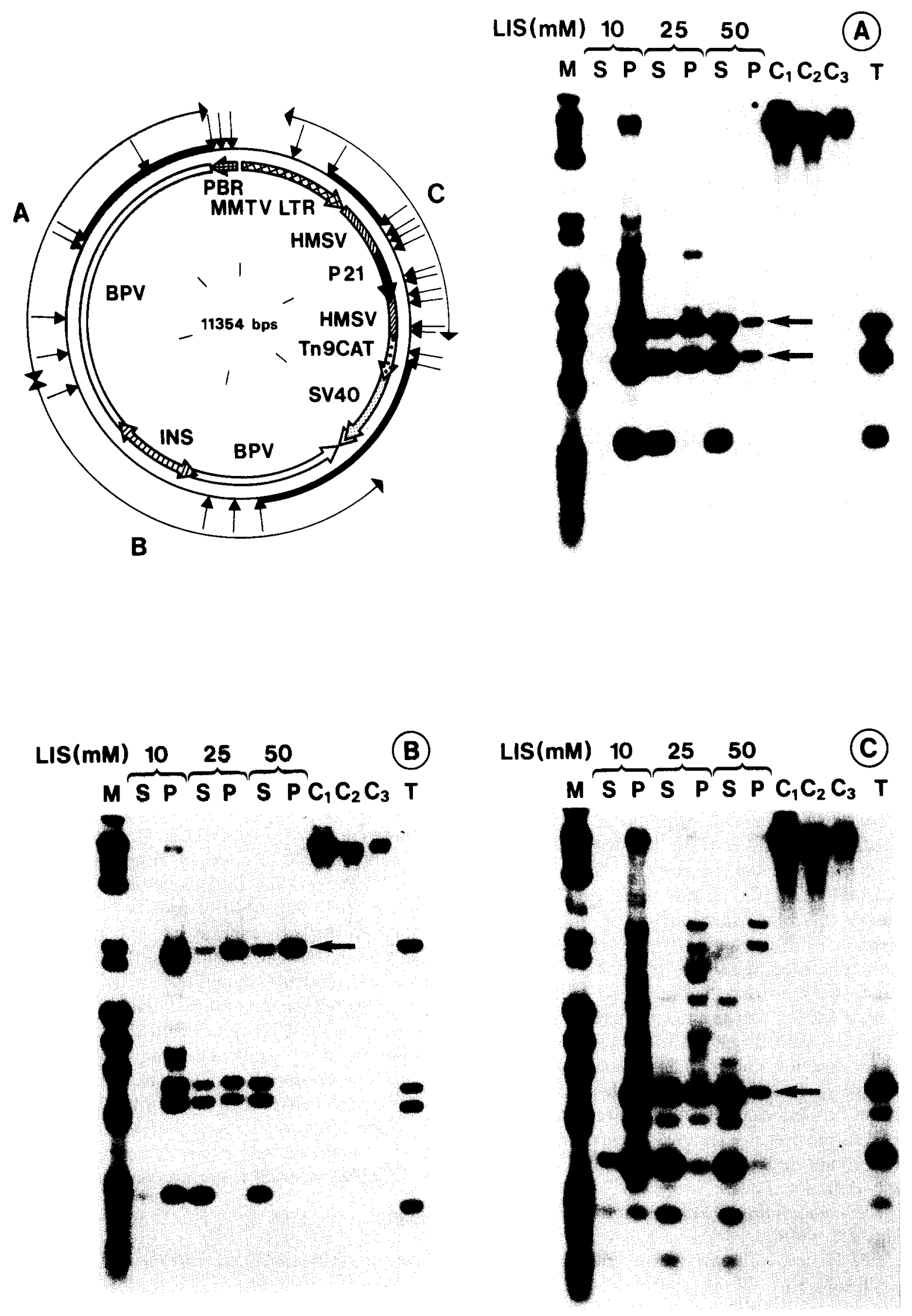


\section{Plasmid construction}

Plasmid pTKFluc contains the reporter gene luciferase under the control of the thymidine kinase (TK) promoter: plasmid pBLCAT2 [38] was cut first with Xhol, then recut with $N d e I$ after filling the XhoI protruding-end with Klenow. The NdeI/XhoI fragment (185 to 601 of pBLCAT2), containing the TK promoter, was isolated and inserted into plasmid plucDSS (luciferase gene) [37] between the HindIII (filled with Klenow) and NdeI sites. Plasmid pVlucl was obtained by insertion of the end-filled Bam HI/SmaI fragment from the Harvey murine sarcoma virus ( 410 to 1011 of HMSV) in the sense orientation upstream of the luciferase gene in plasmid plucDSS. Constructs pTKVLluc1 and pTKVLluc2 are plasmid pTKFluc with the BamHI/SmaI fragment from HMSV inserted in both orientations upstream of the TK promoter. The Bam $\mathrm{HI} / S m a I$ fragment (endfilled at $B a m \mathrm{HI}$ ) was inserted at the SmaI site in pSP72, generating plasmid pSVL. The $B a m \mathrm{HI} / B g l \mathrm{II}$ fragment of this plasmid (containing the HMSV fragment and a piece of the flanking polylinker from pSP72) was inserted at the Bam HI site of plasmid pTKFluc in sense and anti-sense orientations, generating plasmid pTKVLlucl and pTKVLluc2, respectively. Plasmid pTKVLluc3 was obtained by insertion of the NdeI/Xhol fragment from pBLCAT2 into pVLlucl between sites NdeI and Xhol. It contains the Bam HI/SmaI fragment of HMSV inserted between the TK promoter and the luciferase gene in the sense orientations.

Chimeric DNA construct / nuclear scaffold interaction: in situ studies

Nuclei and nuclear scaffolds preparation. Procedures were according to Ref. 9 with the following modifications: $10^{8}$ nuclei were homogenized in $12 \mathrm{ml}$ of lysis buffer $(3.5 \mathrm{mM}$ Tris- $\mathrm{HCl}, 20 \mathrm{mM} \mathrm{KCl}, \mathrm{pH} 7.4,0.05$ $\mathrm{mM}$ spermine, $0.125 \mathrm{mM}$ spermidine, $0.5 \mathrm{mM}$ EDTA, $1 \%$ thioglycerol, $0.1 \%$ digitonin, $100 \mathrm{KIU} / \mathrm{ml}$ aprotinin and $0.1 \mathrm{mM}$ phenylmethylsulfonylfluoride). Ten $A_{260}$ units of nuclei were resuspended in $100 \mu l$ of the same buffer without EDTA and the nuclei were 'stabilized' [9] by incubation with $0.5 \mathrm{mM} \mathrm{CuSO}_{4}$ for $10 \mathrm{~min}$. at $4^{\circ} \mathrm{C}$. Histone depleted nuclei (nuclear scaffolds) were obtained by extraction of the nuclei with various concentrations of 3,5-diiodosalicylic acid, lithium salt (LIS), as indicated in the Results section. Nuclear extraction by LIS was monitored using both light and electron microscopy (data not shown).

In situ digestion of the nuclear scaffolds DNA with HaeIII. Nuclear scaffolds were washed with digestion buffer $(5 \mathrm{mM}$ Hepes, $0.25 \mathrm{mM}$ spermidine, $0.125 \mathrm{mM}$ spermine, $20 \mathrm{mM} \mathrm{KCl}, 70 \mathrm{mM} \mathrm{NaCl}, 10 \mathrm{mM} \mathrm{MgCl}$, $0.1 \%$ digitonin and $100 \mathrm{KIU} / \mathrm{ml}$ aprotinin), recovered by centrifugation and resuspended into $4 \mathrm{ml}$ of the same buffer. They were incubated, under mild shaking, with 2000 units of $\mathrm{Hae} I I I$ for $3 \mathrm{~h}$ at $37^{\circ} \mathrm{C}$. At the end of the treatment the DNA released from the nuclear scaffolds was separated from the DNA associated with it by centrifugation. The DNAs from both fractions were purified and the construct fragments generated by the Hae III digestion were identified by Southern blotting [39], after electrophoresis on agarose gels of the DNA samples ( $10 \mu \mathrm{g} /$ lane), using the probes depicted in Fig. 1.

Chimeric DNA construct / nuclear matrix interaction: in vitro studies

Nuclei preparation, nuclear matrix isolation, and assays of DNA binding to nuclear matrix were performed as previously described [40].

Characterization of the chimeric DNA constructs in cell line 1361.5

While this work was in progress, discrepancies between cutting patterns of the chimeric constructs and the published map were noticed. We undertook a complete mapping of the constructs established in cell line 1361.5. Preliminary studies revealed (1) the presence of approx. 200 copies/cell of the construct, as previously described for this type of cell lines [33], and (2) the insertion of a DNA fragment in the BPV region, between $B a m \mathrm{HI}$ and Eco RI sites. The size of the inserted fragment and the precise position of the insertion were determined by submitting total 1361.5 cellular DNA to double digestions with Bam HI and enzymes cutting at increasing distances from this site. The resulting fragments were visualized using a BPV probe extending from Bam $\mathrm{HI}$ to Ava I (positions $445 \mathrm{I}$ to 3408 of BPV). The results show that more than $95 \%$ of these copies are identical and contain a $1.2 \mathrm{~kb}$ DNA fragment, of unknown origin, inserted between positions $2818(B g l \mathrm{I})$ and $2522(S s p \mathrm{I})$ of BPV (respectively positions 6047 and 6340 of pM23). Digestion of total cellular DNA, with enzymes that do not cut the construct or cut it once, indicates that the rearranged copies are tandemly integrated in the host genome in contrast to previously published data [41]. Hirth extraction of episomal DNA confirmed this observation. The integration of the construct in the host genome results probably from the interruption of ORF E2 or E1 (or both) by the insertion of the $1.2 \mathrm{~kb}$ fragment $[42,43]$. Fig. 1 presents the structure of the rearranged construct. It is drawn as a circle, since the break point of the original construct is not precisely known.

\section{Results}

Chimeric DNA interaction with the nuclear scaffolds: in situ studies

Binding of the chimeric construct to the nuclear scaffold has been investigated in situ. The nuclear 
proteins were extracted from $\mathrm{CuSO}_{4}$ 'stabilized nuclei' with increasing concentrations of LIS, and the DNA was digested with Hae III (Fig. 1). DNA fragments associated with the nuclear scaffold or released in the supernatant were separated by centrifugation. The purified DNA fragments were characterized by Southern blotting after hybridization with probes $\mathrm{A}, \mathrm{B}$ and $\mathrm{C}$ depicted in Fig. 1. A, B and $\mathrm{C}$ are autoradiograms of the blots hybridized with probes $\mathrm{A}, \mathrm{B}$ and $\mathrm{C}$, respectively. Without LIS extraction (lanes C1) Hae III cleavage was undetectable and all the DNA was is in the pellet fraction (not shown). Treatment with $10 \mathrm{mM}$ LIS allowed only a partial cut of the DNA as indicated by the presence in the pellet fraction ( $P$ lanes) of large DNA fragments. Very low or undetectable amounts of DNA fragments were present in the supernatant fraction (S lanes). Complete cut of the DNA with Hae III was achieved only with LIS concentrations $\geq 25 \mathrm{mM}$. Panel A shows an experiment in which probe $A$ was used to visualize fragments located between positions 7916 and 11136 of the construct. Two of the fragments (717 and $978 \mathrm{bp}$ ), marked by arrows, were less easily released into the supernatant than the other fragments; a substantial amount remained associated with the pellet fraction after a $50 \mathrm{mM}$ LIS treatment. These two fragments, marked in bold on the map, encompass the non-coding upstream regulatory region (URR) of the BPV. Panel B shows an experiment in which probe $B$ was used to visualize fragments located between positions 3226 and 8256 of the construct. Comparison of the amounts of the fragments in the supernatant and pellet fractions, upon extraction with increasing concentrations of LIS, clearly shows the retention in the matrix fraction of a approx. $2 \mathrm{~kb}$ fragment. This fragment contains sequences from Tn9CAT, SV40 and BPV. Panel $\mathrm{C}$ presents an experiment in which probe $\mathrm{C}$ was used to visualize fragments located between positions 11336 and 3139 of the construct. The autoradiogram shows that a substantial amount of the 757 bp fragment, marked by an arrow, remained in the pellet fraction after extraction with $50 \mathrm{mM}$ LIS, suggesting its interaction with the nuclear matrix. This fragment contains 3 '-end sequences from the MMTV LTR and v-Ha-ras leader sequences from HMSV.

From the results presented in panels $\mathrm{A}$ and $\mathrm{C}$ it cannot be concluded that pellet fractions isolated after a $50 \mathrm{mM}$ LIS extraction are enriched in DNA fragments hybridizing with probes $\mathrm{A}$ and $\mathrm{C}$, respectively, since the intensity of the bands marked with arrows is lower than that of the control total DNA (lanes T).

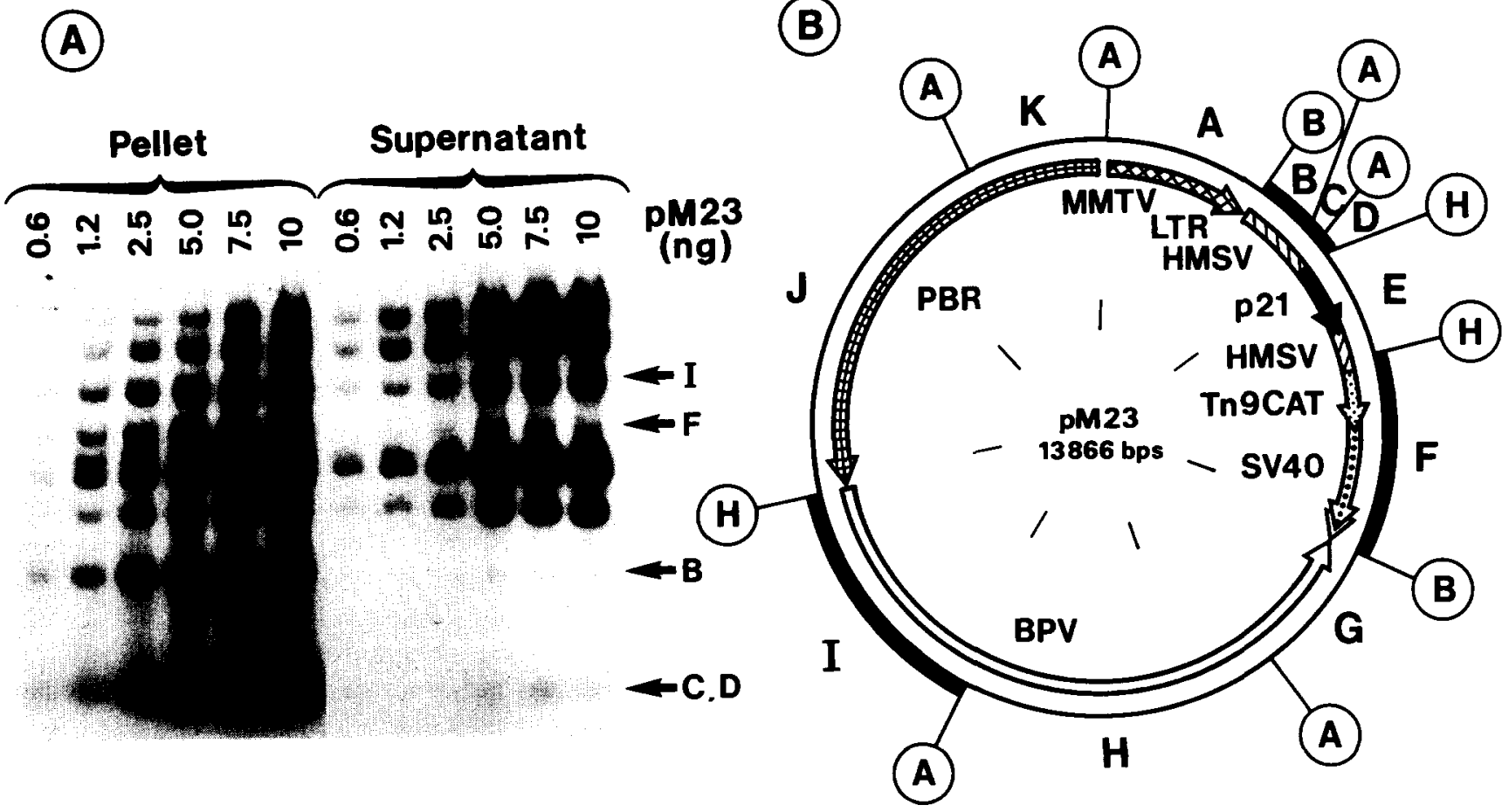

(B)

Fig. 2. In vitro binding of the different regions of $\mathrm{pM} 23$ to the nuclear matrix from 1361.5 cells. (A) Autoradiogram of a gel showing the distribution of the fragments in the pellet and the supernatant fractions. A constant amount of nuclear matrix (corresponding to $5 \cdot 10^{7}$ nuclei) was incubated with increasing amounts of the end-labeled DNA fragments (resulting from the digestion of pM23 with AvaI, Bam HI and $H i n d I I I)$ in the presence of $15 \mu \mathrm{g}$ of competitor $E$. coli DNA. The fragments not (or less) released into the supernatant are marked with an arrow. The letters refer to the fragments presented in B. (B) Map of plasmid pM23. The circled letters indicate the cutting sites used to generate the fragment mixture used in panel A (A, AvaI; B, Bam HI; H, HindIII). The genetic elements of the construct are indicated inside the circle and their labelling is according to Fig. 1. Bold letters indicate the fragments and bold lines highlight fragments interacting with the nuclear matrix. 
However, the corresponding lanes in panel B show two bands of the same size range, which are exclusively found in the S fraction. We therefore conclude that the presence in the $\mathrm{P}$ fraction of the bands marked with arrows in panels $\mathrm{A}$ and $\mathrm{C}$ results from an interaction of the corresponding DNA fragments with the nuclear scaffolds. The lack of enrichment results probably from the interaction with the nuclear scaffold of only a fraction of the DNA fragments population. All together, the results presented in Fig. 1 indicate the binding of three regions of the construct to the nuclear scaffolds.

Chimeric DNA interaction with the nuclear matrix: in vitro studies

An in vitro investigation was performed to get more information on the regions of the chimeric construct which may interact with the nuclear matrix.

In a first experiment (Fig. 2), a constant amount of nuclear matrices isolated from 1361.5 cells was incubated, in the presence of a constant amount of nonspecific $E$. coli competitor DNA, with the indicated amounts of a mixture of end-labeled fragments (generated by the digestion of plasmid pM23 with AvaI, $B a m \mathrm{HI}$ and HindIII (Fig. 2B)). DNA fragments attached to the nuclear matrix (Fig. 2A, 'pellet' lanes) were separated from the free DNA fragments (Fig. 2A, 'supernatant' lanes) by centrifugation. Bands corresponding to fragments $\mathrm{F}, \mathrm{B}, \mathrm{C}$ and $\mathrm{D}$ were exclusively present in the pellet fraction whatever the amount of plasmid pM23 DNA used in the experiment was. This demonstrates the interactions of these 4 fragments with the nuclear matrix. In addition, fragment $I$ was present in the supernatant fraction to a lesser extent than the majority of the other bands. This suggests that fragment I also binds to the nuclear matrix. The complete absence of fragments $F, B, C$ and $D$ in the supernatant, for all the amounts of cleaved pM23 used in the experiment, implies that the nuclear matrix binding sites are high capacity binding sites. The retention, in the pellet fraction, of a limited number of fragments from the mixture demonstrates the existence of a selective binding by the nuclear matrix.

In a second experiment the end-labeled DNA fragments were incubated with nuclear matrices from either 1361.5 or NIH-3T3 cells, in the presence of increasing amounts of sonicated $E$. coli DNA (nonspecific competitor), and the DNA fragments associated with the pellet fraction analyzed. Results presented in Fig. 3 confirm that among all DNA fragments, fragments $\mathrm{I}, \mathrm{F}$ and $\mathrm{B}$ display the strongest interaction with the nuclear matrix in the presence of high amounts of non-specific DNA competitor (60 $\mu \mathrm{g} /$ tube, a 30000 -fold excess). The binding of fragment I was better visualized here than in the previous experiment, and it is clear that fragment $\mathrm{B}$ binds more

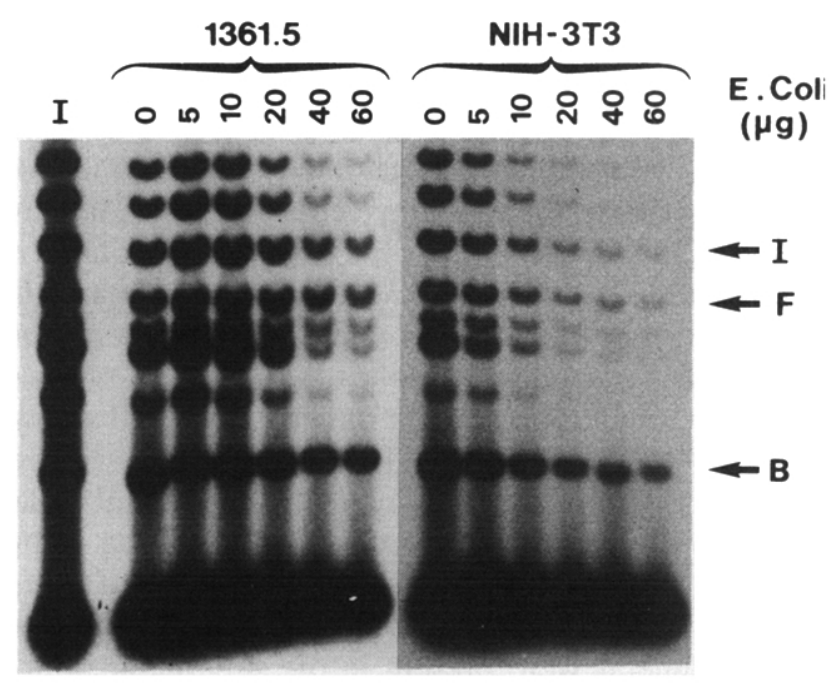

Fig. 3. In vitro comparative binding of the different regions of $\mathrm{pM} 23$ to nuclear matrices from 1361.5 and NIH-3T3 cells. A constant amount $(2 \mathrm{ng})$ of the end-labeled fragment mixture resulting from the digestion of pM23 with Aval, Bam HI, and HindIII (see Fig. 2B) was incubated with nuclear matrices from either 1361.5 or NIH-3T3 cells (equivalent to $5 \cdot 10^{7}$ nuclei), in the presence of the indicated amounts of competitor $E$. coli DNA. The figure shows the autoradiogram of the gel on which have been fractionated the DNAs present in the pellet fraction. The arrows mark the fragments requiring the greatest amounts of $E$. coli DNA to be displaced. Fragments are labeled according to Fig. 2B. Lane I shows the input fragments.

strongly than does either I or F. The similar patterns obtained with nuclear matrices from the two cell lines indicate that the binding of the fragments is not a consequence of the transformation of NIH-3T3 cells by BPV (which might result in the neo-synthesis of viral or cellular proteins associated with the nuclear matrix).

Studies on the interactions of fragment $I$ and various of its sub fragments with the nuclear matrix from 1361.5 cells have been reported elsewhere [40]. Here we have investigated the interaction with the nuclear matrix of the regions located immediately upstream and downstream of the sequences coding for the $\mathrm{v}-\mathrm{Ha}$ ras protein.

Fig. 4 shows the results of an experiment in which a fragment spanning from Bam $\mathrm{HI}$ to HindIII (fragments B, C and D in Fig. 2B) was incubated with nuclear matrices from 1361.5 cells in the presence of increasing amounts of E. coli competitor DNA. A PBR322 fragment that does not bind the nuclear matrix (fragment J, Fig. 2B) was added to the mixture and used as an internal negative control. For amounts of $E$. coli competitor DNA $>20 \mu \mathrm{g} /$ tube, the retention of fragment $\mathrm{J}$ (PBR322) in the pellet fraction was abolished while there was very little change in the binding of fragment 'B-C-D'. A quantitation of the data from the autoradiogram is presented in Fig. $4 \mathrm{C}$. It demonstrates the strong binding of fragment 'B-C-D' to nuclear matrices when assayed in vitro. This fragment was further cut with Acc I (see map on Fig. 4B), and the two resulting 


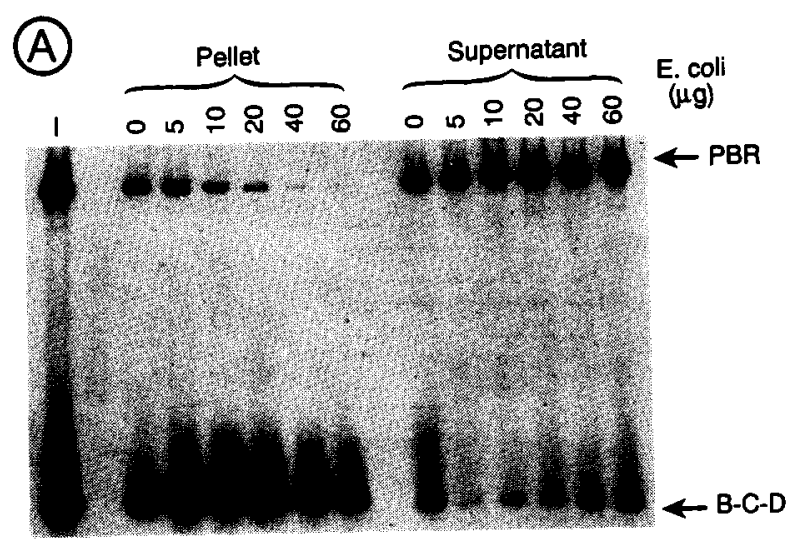

(B)
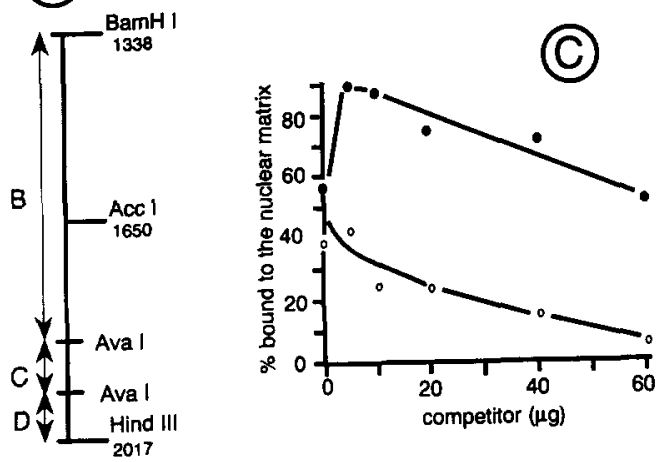

Fig. 4. In vitro binding of the 'B-C-D' region of pM23 to the nuclear matrix from 1361.5 cells. Constant amount of the fragment to be assayed ( $\mathrm{Bam} \mathrm{HI}$ to HindIII), along with a PBR322 fragment (fragment J, Fig. 2A), used as an internal negative control, (0.5 ng each) were incubated with nuclear matrices from 1361.5 cells (equivalent to $5 \cdot 10^{6}$ nuclei) in the presence of the indicated amounts of competitor $E$. coli DNA. A shows the autoradiogram of a gel on which the DNA fragments from the pellet and supernatant fractions were analyzed. Lane I shows the input fragments. The assayed fragment is labeled ' $B-C-D$ ' and its map is shown in B. C shows a quantitation of the data presented in $\mathrm{A}$.

fragments were assayed for their binding to the nuclear matrix (data not shown). They were found to interact equally well, suggesting that the binding of the $B a m \mathrm{HI} /$ HindIII fragment to the nuclear matrix may result from multiple contact points, with one or several proteins.

Fig. 5 presents the results of an experiment in which the interaction of fragment $F$ with the nuclear matrix was investigated. Panel $A$ shows the autoradiogram and panel $B$ a quantitation of the data. They indicate that the full length $F$ fragment spanning from HindIII to Bam HI (labeled $F_{1491}$ ) binds more tightly to the nuclear matrix than does the PBR322 fragment (J) used as a negative control. It also confirms that interaction of the nuclear matrix with fragment $F$ is weaker than with fragment $B$ (compare Fig. $4 \mathrm{C}$ and $5 \mathrm{~B}$ ).

Fig. 6 shows the results of an experiment in which the interaction of $F$ sub-fragments with the nuclear matrix was investigated. Panel A presents the map of
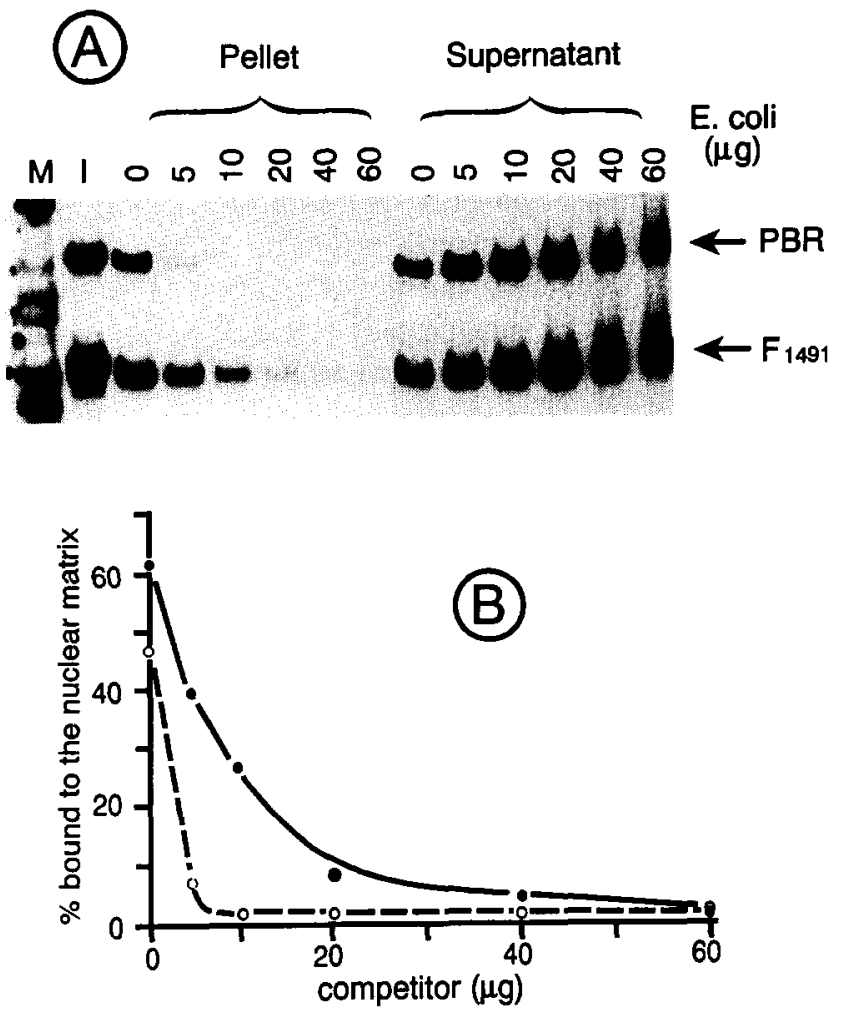

Fig. 5. In vitro binding of the ' $F$ ' region of pM23 to the nuclear matrix from 1361.5 cells. Experimental conditions are identical to those described in Fig. 4 and presentation of the results is the same.

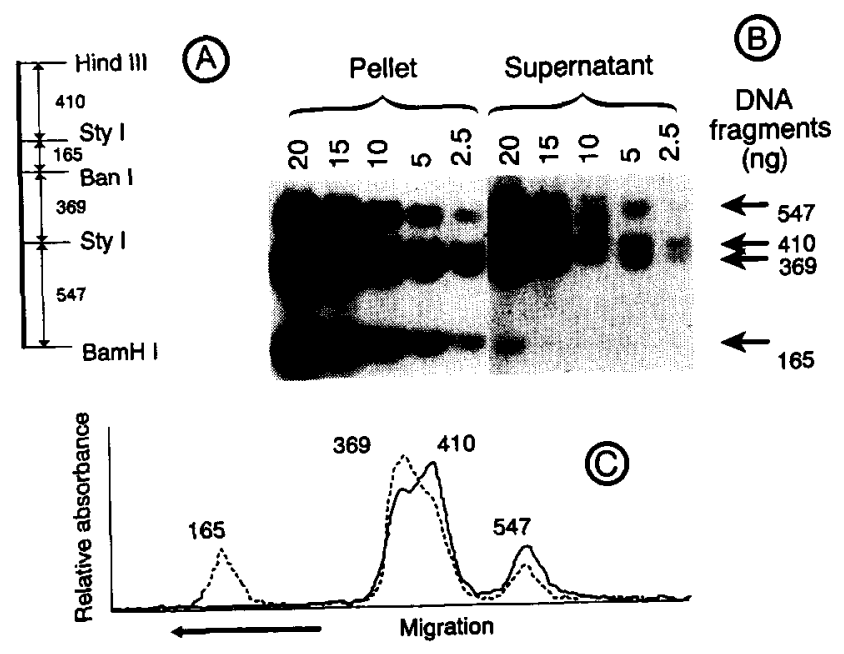

Fig. 6. In vitro binding of DNA fragments covering the ' $\mathrm{F}$ ' region of pM23 to the nuclear matrix from 1361.5 cells. $A$ is a schematic map of the studied region. (B) Autoradiogram of a gel showing the distribution of the fragments in the pellet and the supernatant fractions. A constant amount of nuclear matrix (corresponding to $2 \cdot 10^{7}$ nuclei) was incubated with increasing amounts of the endlabeled DNA fragments (resulting from the digestion of fragment $F$ with Ban I and Sty I) in the presence of $40 \mu \mathrm{g}$ of competitor E. coli DNA. The numbers refer to the size of the fragments presented in A. (C) Densitometric scan of the lanes corresponding to $5 \mathrm{ng}$ of fragments: (- ${ }^{-}$supernatant, $\left(--{ }^{-}\right)$pellet. The absorbance scales have been normalized in order to superimpose the two profiles. 
the fragments. Panel B shows the results of an experiment in which a constant amount of nuclear matrices was incubated with increasing amounts of a mixture of the labelled fragments, in the presence of $40 \mu \mathrm{g} /$ tube of non-specific $E$. coli competitor DNA. This experiment demonstrates a strong retention of the $165 \mathrm{bp}$ Sty I/BanI fragment in the pellet fraction. The other three fragments are substantially released in the supernatant fraction although their distribution between pellet and supernatant varies slightly. This is visible on panel $\mathrm{C}$ that shows a densitometric scan of the two lanes of the autoradiogram corresponding to an incubation with $5 \mathrm{ng}$ of labelled fragments: there is more of the $369 \mathrm{bp}$ fragment in the pellet fraction while more of the $410 \mathrm{bp}$ fragment is found in the supernatant.

All along, these in vitro studies demonstrate the interaction of three regions of the construct with the nuclear matrix. These regions overlap the region shown to interact with the nuclear matrix in the in situ experiments.

\section{Functional studies}

When assayed in vitro, the region of pM23, containing fragments $\mathrm{B}, \mathrm{C}$ and $\mathrm{D}$ displayed the strongest binding to the nuclear matrix and an overlapping fragment was found to interact with the nuclear matrix in in situ experiments. The main structural features of this region are depicted in Fig. 8a. It contains a region that comes from the HMSV which displays $98 \%$ of homology with rat genome repetitive sequences termed 'ID' sequences [44]. Since it is located immediately upstream of coding sequences, we have investigated its effects on the expression of the reporter gene luciferase, driven by the TK promoter, in transient transfections experiments. A DNA fragment spanning from Bam HI to SmaI (fragments B + C in Fig. 2B) was inserted either upstream of the TK promoter or between the promoter and the reporter gene (its natural occurrence in the viral genome). The region spanning fragment $\mathrm{D}$, which contains ras gene leader sequences and the ATG was omitted in these constructs because it may interfere in the expression assay. The results of the experiments are presented in Fig. 7. The insertion of this fragment, in the sense orientation, upstream of the TK promoter (pTKVLluc1) increased significantly the luciferase activity, when compared to a construct containing only the TK promoter driving the luciferase gene (pTKFluc). In contrast, its insertion in the sense orientation between the TK promoter and the luciferase gene (pTKVLluc3) had no significant effect. The ID sequences present within fragment 'B-C' can be transcribed by RNA polymerase III [45] and can act as a positive regulator of RNA polymerase 11 genes in the cells in which they are highly transcribed [46]. In addition, the involvement of repetitive DNA sequences in nuclear matrix attachment has been described in

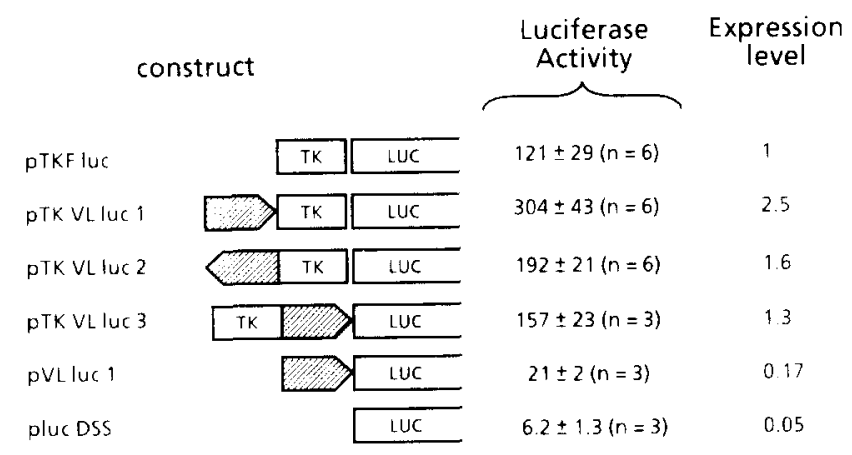

Fig. 7. Transient transfection of NIH-3T3 cells with recombinant DNA constructs, containing the ' $\mathrm{B}-\mathrm{C}$ ' region of pM23 coupled to a heterologous promoter. Schematic maps of the constructs are presented in the left column. TK is the thymidine kinase promoter and LUC the luciferase reporter gene. The ' $\mathrm{B}-\mathrm{C}$ ' region of $\mathrm{pM} 23$ is indicated by the hatched arrow whose direction indicates the orientation of the insert in the construct. Luciferase activities, corrected for transfection efficiencies, are expressed in arbitrary units (see Materials and Methods) and are the mean of the values obtained from three to six independent transfections.

other systems [47]. RNA synthesis from such a promoter or from promoters located within this fragment was tested: a plasmid containing the luciferase gene with the 'B-C' fragment inserted upstream of it, in the sense orientation ( $p$ VLluc1), was assayed. Luciferase activity was much lower than for the control pTKFluc, indicating that such a promoter within the 'B-C' region of the construct does not significantly contribute to the activity of the TK promoter in plasmid pTKVLluc1. In addition, since the enhancement of the TK promoter activity is not observed when the fragment is inserted downstream of it, a positive regulator effect of ID sequences on polymerase II activity seems unlikely in these experiments. The establishment of the constructs in permanent cell lines will allow to assess the significance of the results presented here.

\section{Discussion}

In this paper we report studies on the interaction with the nuclear matrix and scaffold of a chimeric DNA construct containing a hormonally regulated transcription unit (MMTV LTR driving v-Ha-ras) linked to the $69 \%$ transforming fragment of the BPV genome. These studies were performed in cell line 1361.5 that contains 200 copies/cell of the above described construct, tandemly integrated. Two approaches were used: low-salt chromatin extraction of 'stabilized' nuclei (LIS extraction) [9] followed by an in situ cleavage of the nuclear scaffold DNA with HaeIII, and in vitro interaction of nuclear matrices prepared from 1361.5 and NIH-3T3 cells using a high salt extraction procedure [20], with end-labeled DNA fragments covering all the construct. We used two experimental procedures because the nuclear matrix protein compo- 
sition depends largely on the isolation conditions, making its definition mostly operational. It is therefore possible, that different methods of analysis detect somewhat a different spectrum of matrix-attached DNA fragments [25]. The low-salt LIS extraction procedure, used here for in situ experiments, involves a nuclei 'stabilization' step achieved either by heat treatment or addition of $\mathrm{Cu}^{2+}$ [9]. This stabilization process, that allows strong interactions between components of the nuclei, is still poorly understood and may lead to protein oxidation resulting in a change of their binding properties. The high salt nuclear matrix preparation [20], used here for the in vitro studies, do not involve such a 'stabilization' of the nuclei. As a consequence untreated nuclei may, during the salt extraction, release some of the proteins that are retained in the nuclear matrix fraction upon 'stabilization'. In addition non-specific trapping of protein complexes in the matrix fraction, resulting of the high salt extraction, has been described $[9,48]$. Furthermore, in vitro studies that allow to vary the DNA/matrix stoichiometry may result in a better visualization of their interaction.

The two types of experiments demonstrate the binding of three regions of the chimeric construct, present in 1361.5 cells, to the nuclear matrix. However, there are differences in the strength of interaction and, at least for one region, in their boundaries. Results are summarized in Fig. 8. For each region features characteristic of SAR/MAR sequences are shown: the \% AT is plotted on top of the panel and vertical arrows point out the topoisomerase II consensus. A description of the region is presented underneath the map and the fragments interacting with the nuclear matrix in each type of experiment are marked.

The region of the construct described in panel a encompasses fragments B, C and D (see Fig. 2B). It contains approx. 300 bp of the 3'-end of MMTV LTR and approx. $700 \mathrm{bp}$ of HMSV v-Ha-ras leader sequences. This region does not show a typical SAR/MAR structure [49]. It contains clusters of AT rich regions, but only a few scattered Topoisomerase II consensus sequences. The in vitro studies demonstrated a strong binding of the Bam HI/HindIII HMSV fragment and the absence of MMTV LTR binding to nuclear matrices from both 1361.5 and NIH-3T3 cells. The strong in vitro interaction of the HMSV fragment with the nuclear matrix contrasts with its behavior in in situ studies. The Hae III/Hae III fragment is clearly retained in the pellet fraction, but significant amounts are released in the supernatant fraction for LIS concentrations above $25 \mathrm{mM}$, suggesting a much weaker interaction. This may result from differences in matrix composition, as previously mentioned. A non-specific trapping of proteins, due to the high-salt extraction procedure, may contribute to the strong matrix/HMSV fragment interaction observed in vitro. Conversely, the relatively low percentage of the fragment bound to the nuclear scaffolds in the in situ experiments may result from an alteration of the binding properties of some

(a)

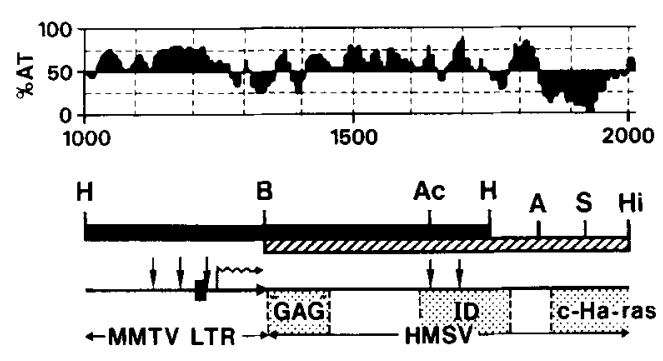

(b)

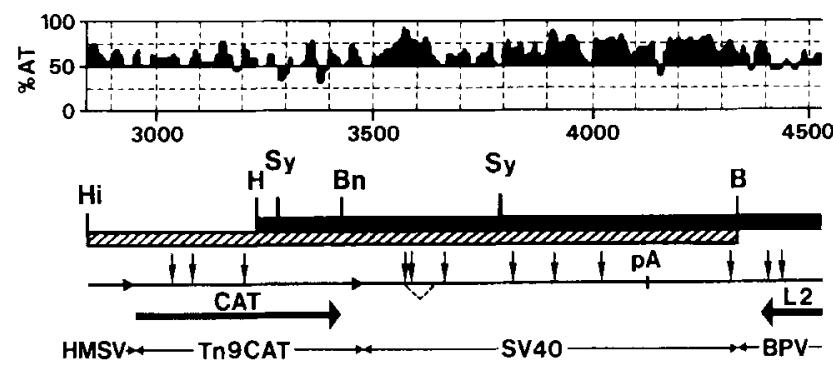

(c)

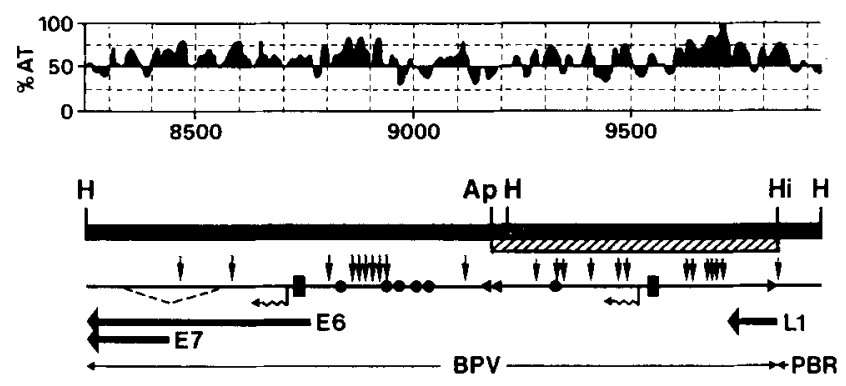

Fig. 8. Comparison of the three regions of the construct pM23 interacting with the nuclear matrix. Panels $a, b$, and $c$ present regions encompassing fragments B-C-D, F, and I (depicted in Fig. 2B), respectively. The graph on top of each panel shows the \% AT through a 20 bp window. Numbers underneath refer to the numbering of pM23D (Fig. 2B). A map of each region is presented under the graph. The fragments retained in the nuclear matrix fraction in the in situ studies are figured in bold and the fragments interacting in vitro with high-salt nuclear matrices are figured hatched. The letters on top are the restriction enzyme cutting sites (A, AlaI; Ac, AccI; Ap, ApaLI; B, Bam HI; Bn, BanI; H, Hae III; Hi, HindIII; Sy, $S t y$ I). For each region the genetic elements are figured. Closed boxes are TATA boxes, closed circles are binding sites for the BPV E2 transactivator, closed triangles mark a direct repeat, dashed lines indicate splice sites, small horizontal lines with an arrow are RNA start sites, vertical arrows on top of the line indicate topoisomerase II consensus sequences ( $70 \%$ homology with drosophila consensus sequence), and $\mathrm{pA}$ is a polyadenylation signal. Bold lines ending with an arrow, at the bottom of each panel, indicate open reading frames. On panel a, the grey areas are regions homologous to viral GAG leader sequences, ID sequences and exon -1 from c-Ha-ras. On panel b, the region spanning from position 4500 to 5509 ( Hae III site) has been cut. 
proteins during the 'stabilization' step. It may also result from the involvement, in a dynamic process, of only a fraction of the construct population at the time. Transient transfection studies indicated that this region is able to moderately enhance the TK promoter activity, when located upstream of it in the sense orientation.

Panel $b$ shows the region of the construct encompassing fragment $F$ (see Fig. 2B), which is located downstream of the $\mathrm{v}$-Ha-ras coding region. It contains sequences from Tn9CAT, SV40 (splice and polyadenylation sites), and BPV (end of the early region and beginning of the late region). This region is moderately AT rich, except for the SV40 sequences, and contains a few scattered topoisomerase II consensus sequences. In situ digestion of nuclear scaffolds demonstrate the strong interaction with the nuclear matrix of a large 2.2 kb HaeIII/Hae III fragment. The BPV sequences present within this fragment ( $\mathrm{HaellI} / \mathrm{Bam} \mathrm{HI}$ region) do not contribute significantly to the interaction. Similar experiments performed using ID13 cells (a transformed clone of the $\mathrm{C} 127$ mouse cell line carrying approx. 150 copies per cell of wild-type BPV [50]) failed to detect any binding to the nuclear scaffolds of the BPV fragment spanning this region (data not shown). The large size of this Hae III/Hae III fragment, may contribute to its preferential retention in the pellet fraction, but cannot completely account for it since, in experiments in which a partial digestion of the construct occurred, fragments over $2 \mathrm{~kb}$ were found mostly in the supernatant fraction, while smaller ones were detected exclusively in the pellet fraction (data not shown). In vitro studies demonstrate the binding of a $165 \mathrm{bp}$ fragment, located within the Tn9CAT region which overlaps the 5 '-end of the Hae III/Hae III fragment suggesting that the region interacting with the nuclear matrix is contained within the HaeIII/Ban Il region. The biological significance of the binding of bacterial Tn9CAT sequences to eukaryotic nuclear matrices remains under question.

Panel $\mathrm{c}$ presents the third region involved in the interaction with nuclear matrices. It encompasses fragment I (see Fig. 2B). Interestingly this region contains the BPV upstream regulatory region (URR). In this region are located the PMS-1 region [51] (responsible of plasmid state maintenance of the virus), the viral origin of replication [52], two promoters [53] and an enhancer transactivated by the viral E2 protein [54,55]. Its structure resembles a MAR/SAR structure, with clusters of AT rich regions (up to approx. $100 \%$ around position 9700 of the construct) and clusters of topoisomerase II consensus sequences. The in situ experiments show the binding to the nuclear matrix of two contiguous HaeIII/Hae III fragments. These two fragments show an identical behavior, upon treatment of the nuclei with increasing concentrations of LIS, with only approx. $10 \%$ of the amount of each fragment remaining associated with the nuclear matrix at the highest LIS concentration used. This may be due to the involvement of a limited number of viral origins of replication at the time in the replication process (as described for the virus in ID13 cells [47]). The in vitro experiments presented here and a more detailed analysis previously reported [40] allow to localize the region interacting with high-salt nuclear matrices within a fragment spanning from ApaLI to HindIII. This fragment contains the BPV PMS-1 region and superimposes almost exactly with one of the two Hae III/Hae III fragments binding to the nuclear matrix in the in situ experiments. It is adjacent to the other Hae III/Hae III fragment that contains the viral origin of replication and most of the E2 transactivated enhancer.

The differences in binding to nuclear matrices and scaffolds of the PMS- 1 and core replication origin of the BPV suggest that different sets of proteins may be involved in the interaction. This is of importance, since it has been recently proposed that PMS-1 could be part of a composite replication origin [52]. This region could provide information at a distance and be required for effective replication initiation. An increasing number of reports describes the interaction of replication origins with the nuclear matrices. However in higher eukaryotes, data are difficult to interpret accurately, since a precise localization of the origins of replication has not been achieved. In this regard, the BPV, with an origin of replication localized within a $200 \mathrm{bp}$ region, is a system of choice to investigate the role of the nuclear matrix in DNA replication.

\section{Acknowledgments}

We are very grateful to S. Subramani for plasmid pSV0AL-delta5'Luc and to G.L. Hager and M.C. Ostrowski for cell line 1361.5. D. Fortin provided us with a very efficient technical help. C. Legris and J.C. Lambert helped us with the art work. This work was partially supported by the Centre National de la Recherche Scientifique, Association pour la Recherche sur le Cancer, and Section de Paris de la Ligue Contre le Cancer. F.G. is a fellow of l'Association pour la Recherche sur le Cancer.

\section{References}

1 Benyajati, C. and Worcel, A. (1976) Cell 9, 393-407.

2 Cook, P.R. and Brazell, I.A. (1976) J. Cell Sci. 22, 287-302.

3 Paulson, J.R. and Laemmli, U.K. (1977) Cell 12, 817-828.

4 Vogelstein, B., Pardoll, D.M. and Coffey, D.S. (1980) Cell 22, $79-85$.

5 Georgiev, G.P. and Chentsov, Y.S. (1960) Proc. Acad. Sci. USSR 132, 199-202.

6 Berezney, R. and Coffey, D.S. (1976) Adv. Enzyme Regul. 14, $63-100$. 
7 Herman, R., Weymouth, L. and Penman, S. (1978) J. Cell Biol. 78, 663-674.

8 Cook, P.R. and Brazell, I.A. (1980) Nucleic Acids Res. 8, 28952906.

9 Mirkovitch, J., Mirault, M.-E. and Laemmli, U.K. (1984) Cell 39, 223-232.

10 Elgin, S.C.R. (1990) Current Opin. Cell Biol. 2, 437-445.

11 Razin, S.V., Kekelidze, M.G., Lukanidin, E.M., Scherrer, K. and Georgiev, G.P. (1986) Nucleic Acids Res. 14, 8189-8207.

12 Berezney, R. and Coffey, D.S. (1975) Science 189, 291-293.

13 Dijkwel, P.A., Wenink, P.W. and Poddighe, J. (1986) Nucleic Acids Res. 14, 3241-3249.

14 Jackson, D.A. and Cook, P.R. (1986) EMBO J. 5, 1403-1410.

15 Robinson, S.I., Small, D., Rejeau, I., McKnight, S.G. and Vogelstein, B. (1983) Nucleic Acids Res. 11, 5113-5131.

16 Ciejek, E.M., Tsai, H.H. and O'Malley, B.W. (1983) Nature 306, 607-609.

17 Small, D., Nelkin, B. and Vogelstein, B. (1985) Nucleic acids res. 13, 2413-2431.

18 Zeitlin, S., Parent, A., Silverstein, S. and Efstratiadis, A. (1987) Mol. Cell. Biol. 7, 111-120.

19 Razin, S.V. (1987) BioEssays, 6, 19-23.

20 Cockerill, P.N. and Garrard, W.T. (1986) Cell 44, 273-282.

21 Phi-Van, L. and Stratling, W.H. (1988) EMBO J. 7, 655-664.

22 Gasser, S.M., Amati, B.B., Cardenas, M.E. and Hofmann, J.F.-X. (1989) Int. Rev. Cytol. 119, 57-96.

23 Kalandadze, A.G., Bushara, S.A., Vasseytzky, Y.S. and Razin, S.V. (1990) Biochem. Biophys. Res. Commun. 168, 9-15.

24 Farache, G.F., Razin, S.V., Rzeszowska-wolny, J., Moreau, J., Recillas Targa, F. and Scherrer, K. (1990) Mol. Cell. Biol. 10, 53-49-5358.

25 Dijkwel, P.A. and Hamlin, J.L. (1988) Mol. Cell. Biol. 8, 53985409.

26 Avramova, Z. and Paneva, E. (1992) Biochem. Biophys. Res. Commun. 182, 78-85.

27 Berrios, M., Osheroff, N. and Fisher, P.A. (1985) Proc. Natl. Acad. Sci. USA 82, 4142-4146.

28 Earnshaw, W.C., Halligan, B., Cooke, C.A., Heck, M.M.S. and Liu, L.F. (1985) J. Cell Biol. 100, 1706-1715.

29 Gasser, S.M. and Laemmli, U.K. (1986) Cell 46, 521-530.

30 Hofmann, J.F.-X., Laroche, T., Brand, A.H. and Gasser, S.M. (1989) Cell 57, 725-737.
31 von Kries, J.P., Buhrmester, H. and Stratling, W.H. (1991) Cell $64,123-135$.

32 Richard-Foy, H. and Hager, G.L. (1987) EMBO J. 6, 2321-2328.

33 Ostrowski, M.C., Richard-Foy, H., Wolford, R.G., Berard, D.S. and Hager, G.L. (1983) Mol. Cell. Biol. 3, 2045-2057.

34 Gross, D.S. and Garrard, W.T. (1987) Trends Biochem. Sci. 12, 293-297.

35 Graham, F.L. and Van der Eb, A.J. (1973) Virology 52, 456-467.

36 Nguyen, V.T., Morange, M. and Bensaude, O. (1988) Anal. Biochem. 171, 404-408.

37 Gouilleux, F., Sola, B., Couette, B. and Richard-Foy, H. (1991) Nucleic Acids Res. 19, 1563-1569.

38 Lucklow, B. and Schutz, G. (1987) Nucleic Acids Res. 15, 5490.

39 Southern, E.M. (1975) J. Mol. Biol. 98, 503-517.

40 Adom, J. and Richard-Foy, H. (1991) Biochem. Biophys. Res. Commun. 176, 479-485.

41 Ostrowski, M.C. (1987) Nucleic Acids Res. 15, 6957-6971.

42 Sarver, N., Rabson, M.S., Yang, Y.C., Byrne, J.C. and Howley, P.M. (1984) J. Virol. 52, 377-388.

43 Lusky, M. and Botchan, M. (1984) J. Virol. 53, 955-965.

44 Sutcliffe, J.G., Milner, R.J., Bloom, F.E. and Lerner, R.A. (1982) Proc. Natl. Acad. Sci. USA 79, 4942-4946.

45 Sutcliffe, J.G., Milner, R.J., Gottesfeld, J.M. and Lerner, R.A. (1984) Nature 308, 237-241.

46 McKinnon, R.D., Shinnick, T.M. and Sutcliffe, J.G. (1986) Proc. Natl. Acad. Sci. USA 83, 3751-3755.

47 Farache, G., Razin, S.V., Recillas Targa, F. and Scherrer, K. (1990) Nucleic Acids Res. 18, 401-409.

48 Kirov, N., Djondjurov, L. and Tsanev, R. (1984) J. Mol. Biol. 180, 601-614.

49 Gasser, S.M. and Laemmli, U.K. (1986) EMBO J. 5, 511-518.

50 Turek, L.P., Byrne, J.C., Lowy, D.R., Dvoretzky, I., Friedman, R.M. and Howley, P.M. (1982) Proc. Natl. Acad. Sci. USA 79, 7914-7918.

51 Lusky, M. and Botchan, M. (1984) Cell 36, 391-401.

52 Yang, L. and Botchan, M. (1990) J. Virol. 64, 5903-59115.

53 Stenlund, A., Bream, G.L. and Botchan, M. (1987) Science 236, 1666-1671.

54 Spalholz, B.A., Yang, Y.C. and Howley, P.M. (1985) Cell 42, 183-191.

55 Androphy, E.J., Lowy, D.R. and Schiller, J.T. (1987) Nature 325, 70-73. 